\title{
Esperando a Godot, todavía...
}

\author{
Waiting for Godot, still...
}

José Miguel Rodríguez Zamora



Esta obra está bajo una licencia Creative Commons Reconocimiento-No comercial-Sin Obra Derivada 


\title{
Esperando a Godot, todavía... Waiting for Godot, still...
}

\author{
José Miguel Rodríguez Zamora ${ }^{1}$ \\ Universidad de Costa Rica \\ Costa Rica
}

Recibido: 02 de marzo de 2017 Aprobado: 08 de junio de 2017

\section{Resumen}

Este artículo revisa la relación que existe entre la obra de Samuel Beckett, Esperando a Godot, con el contexto filosófico. El llamado teatro del absurdo representa el sentir de una época determinada, pero Beckett consigue traspasar los límites del tiempo para crear una obra de importancia universal con un profundo significado para nuestros días.

Palabras clave: Beckett; teatro del absurdo; Esperando a Godot; existencialismo

\begin{abstract}
This article reviews the relationship between the work of Samuel Beckett, Waiting for Godot, within the philosophical context. As it's called, the theater of the absurd represents the feelings of a certain era but Beckett continues to transcend the limits of time to create a piece of universal importance with deep meaning in our day.
\end{abstract}

Keywords: Beckett; theater of the absurd; Waiting for Godot; existentialism

1 Máster en Filosofía por la Universidad de Costa Rica (UCR). Profesor jubilado de la UCR. Correo electrónico: jomiroz@gmail.com 


\author{
Estragón: ¿Y qué haremos ahora? \\ Vladimir: No sé. \\ Estragón: Vayámonos. \\ Vladimir: No podemos. \\ Estragón: ¿Por qué? \\ Vladimir: Esperamos a Godot. \\ Estragón: Es cierto.
}

(Beckett, 2006, p.164)

\title{
Caminar hacia la noche
}

¿Por qué el teatro de Beckett sigue representándose? ¿Cuál es la razón de que aún tenga interés? No es fácil encontrar una respuesta sencilla a tales preguntas. Esperando a Godot (1952) es un texto engañosamente simple que sobrepasa las categorías del teatro del absurdo, la pieza existencial o el minimalismo teatral. ¿Cómo penetrar en su irreal realidad? Muchos caminos pueden ser posible. Aquí se proponen varias líneas de reflexión que permiten una mayor comprensión del texto. No se trata de una discusión sobre las propuestas o sobre el teatro de Beckett; más bien es una aproximación funcional que señala varios elementos filosóficos presentes en la obra. En un primer término conviene revisar la compleja época en la cual fue escrito, ¿paralela a la nuestra?, para luego considerar la importancia que posee el contexto filosófico para la comprensión de esta famosa obra de teatro.

La oposición entre el sentimiento del absurdo y la idea de que la existencia tiene un sentido suscitó un debate permanente entre los intelectuales occidentales a lo largo del siglo $X X$. Tuvo su culminación en Europa después de la Segunda Guerra Mundial. La desolación producida por esa conflagración motivó que se reflexionara sobre el valor de la vida, considerada tanto en la existencia personal e individual como sobre el sentido de la comunidad humana. No sorprende que después de tal conflicto hubiera un sentimiento de fracaso, impotencia y sinsentido frente a un panorama devastador y confuso, aún en parte de las naciones vencedoras. Y no podría ser de otra forma: se había roto, para siempre, el optimismo racionalista que predominó durante el siglo XIX entre las clases dominantes del occidente capitalista. Ahora el ser humano, desolado por los horrores de dos guerras mundiales, "camina diariamente hacia el interior de la noche" como había advertido Heidegger (2003).

Son los años de la Guerra Fría que se inicia poco después de la Segunda Guerra Mundial. En este período se produce la aparición de diversos marxismos y, a la vez, de sus crisis, del psicoanálisis, de los procesos de descolonización, principalmente en África y el sudeste asiático, y es la época también de recoger los restos de la experimentación vanguardista surgidos a principios del siglo. Es la época durante la cual regresan los exiliados y se reconfigura la cultura europea occidental. Ante el horizonte yermo de la reconstrucción europea, el cuestionamiento sobre la cultura derivó hacia una pregunta mucho más 
profunda: el significado de la vida. Los intelectuales y los artistas recogieron este sentimiento en sus obras (Kandel 2012). El teatro, la literatura, la música, la pintura, y en general, todas las artes, y aún en gran parte la propia filosofía, fueron una expresión de esta época dramática caracterizada por la pérdida de los fundamentos tradicionales (Benjamin 2013).

Ciertamente, cada pensamiento es fruto de su época. Surge el existencialismo de Sartre, las denuncias de Simone de Beauvoir y de Camus, el nihilismo de Cioran, la poesía de Celan, o las ensoñaciones ante la muerte del Virgilio de Broch; también Malraux explora la tragedia de la condición humana y Saint-Exupéry propondrá una nueva y mordaz ciudadela muy lejos de El Principito. Por supuesto, el teatro de Beckett, lonescu, Harold Pinter, Edward Albee, Dürrenmatt, entre otros, expresarán este mal du siècle. Asimismo, Büchner lo había denunciado décadas antes en su obra teatral Woyzeck de claro corte expresionista, magistralmente llevada al plano de la ópera por Berg y al cine por Herzog. Y Bertolt Brecht, por su parte, había confrontado a la sociedad burguesa con el absurdo del poder, de la exclusión y de la violencia con un teatro de fuerte crítica social (Nussbaum, 2008). En el teatro de Beckett no están los lamentos de Antígona, Casandra, Laertes, Edipo o Heracles; no habrá un "escenario de los horrores" (Kaufmann 1978, p. 474), como bien calificó Walter Kaufmann al teatro del absurdo. Ahora, si se quiere, hay un desplazamiento del horror hacia el sinsentido, la angustia y la náusea existencial. Es la reconfiguración en la historia de la literatura de una concepción de la épica y de la tragedia en una esfera que redefine, además, la propia estética (Moretti, 1996, pp. 182-213).

Desde el período comprendido entre las dos guerras mundiales -y seguramente en la década de 1920 - aparecen varias manifestaciones importantes tanto en Europa, Estados Unidos como en las corrientes vanguardistas de América Latina. Una de ellas es la revista francesa Les Temps Modernes como baluarte expresivo de tal movimiento. El llamado Nouveau Roman se hará eco de estas obras dramáticas y literarias para construir un texto sin héroes, estático y carente de significado. Y no podría faltar el cine: recoge el sentimiento de esta época en obras tan destacadas como las sucesivas adaptaciones de El extranjero y La peste de Albert Camus, los cortos de Ripstein, varias obras de Buñuel y las puestas en escena de A puerta cerrada y de otras obras teatrales de Sartre. Samuel Beckett será uno de los más célebres representantes de esta corriente, en sus obras supo captar el sentimiento de desolación, impotencia y, a la vez, de un humor desesperanzado.

\section{Absurdo y filosofía}

¿Tiene la vida algún significado? ¿Vale la pena esta existencia? O, por el contrario, todo es ilusorio e insensato. Es frecuente que a lo largo de la historia el ser humano se enfrente a estas cuestiones, sobre todo en situaciones límites. Y esto fue lo que ocurrió cuando 
los intelectuales se encontraron ante una Europa destruida y un mundo que buscaba nuevas vías de organización. Definitivamente, el sueño de la razón había producido monstruos.

¿Teatro del absurdo? Ciertamente. Sin embargo, conviene hacer una precisión oportuna. La palabra absurdo puede ser engañosa. Por una parte, parece que posee una connotación indicando lo irracional, inadecuado, extravagante y extraño; sin embargo, está cargada de muchos matices polisémicos de gran profundidad filosófica y psicológica. Este vocablo se compone del prefijo latino ab correspondiente a la preposición de y de la palabra sordus (sordo). Y se le solía aplicar al ámbito musical para indicar la incapacidad de escuchar o de no escuchar bien y, además, de disonante. Luego pasó a significar inoportuno e inepto (Segura Munguía, 1985, p. 7).

Puede afirmarse que las raíces de la filosofía del absurdo se remontan a Schopenhauer, Nietzsche, Kafka y Kierkegaard. Y en parte es cierto, sin embargo, su origen filosófico es mucho más antiguo porque puede encontrarse ya en Cicerón (Sobre el orador, 2002). En estos casos, el absurdo no es correlativo de sinsentido sino, más bien, remite a una dimensión cognoscitiva y existencial diferente y alternativa. Aquí se aprecia un primer elemento significativo del absurdo. Más adelante, esta filosofía va a subrayar las contradicciones de una sociedad burguesa conformista e ingenuamente optimista. Pues ya no se trataba únicamente de las denuncias de las dramáticas condiciones sociales y políticas propias de obras como las de Dickens o Zola, sino algo mucho más profundo y radical: cuestionar la existencia misma. De esta manera, el absurdo, cargado de elementos negativos e incluso vejatorios, adquiere una dimensión filosófica y psicosocial. Recuérdese que estos elementos fundantes ya se encontraban tanto en Nietzsche como en Schopenhauer. Terry Eagleton lo resume de la siguiente forma:

La existencia humana, tanto para Schopenhauer como para el joven Nietzsche, es un campo de dolores atroz. Pero mientras que para Schopenhauer extrae de ello la lección sofóclea de que sería mejora no haber nacido, y de hecho lo piensa así para las masas afanadas de la historia, el vuelo de Nietzsche sobre el espanto no se dirige hacia la inexistencia, sino hacia la metamorfosis trágica (Eagleton, 2011, p. 95).

Sin embargo, Nietzsche aprende a aceptar el sufrimiento y lo transforma en afirmación vital. Este paso, ¿lo dará Beckett? Ciertamente, era el fin radical de una época. Pero, si todo termina, a la vez todo tendrá que volver a empezar. Pero, ¿cómo? Propiamente en el siglo XX, la obra de Jean-Paul Sartre, en particular su tratado de ontología existencial El ser y la nada, sus obras de teatro y sus novelas, representaron un síntoma de la época. Este combativo filósofo francés destacó lo absurdo de la libertad humana que implica una elección siempre desde la nada, resumido en una famosa frase: "el hombre está condenado a ser libre” (Sartre 1998, pp.11-12). Con este oxímoron, el pensador francés quería acentuar 
lo insensato de la existencia desde la propia condición humana inmersa en un mundo de sinsentido e irracionalidad. Un mundo en el cual cada hombre y cada mujer debe tomar decisiones continuamente y, como es sabido, elegir también significa renunciar; en ocasiones, incluso a desistir de lo que más se ama. Según el filósofo francés, toda elección carece de apoyo y, por lo tanto, se halla más allá de todas las razones. Como los personajes de Esperando a Godot, el ser humano está desamparado, vive a la intemperie. Enclavado dentro de un tiempo y de un espacio en el cual debe hacerse a sí mismo, pero sin encontrar en su interior una guía espiritual y, mucho menos, apoyarse cínicamente en una moral externa, ya sea esta religiosa, política o simplemente utilitaria.

De ahí que cada uno sea responsable absoluto de sus propios actos. ¿Para qué? Al fin de cuentas el "hombre es una pasión inútil" (Sartre, 1966, p.747). Aquí reside el origen de la angustia y es el umbral de este humanismo ateo. Pero, en consecuencia, la convivencia con los otros se vuelve insoportable. En especial, la mirada del otro que se convierte en permanente inquisición y tortura. "El infierno son los otros" ha dicho en una de sus obras teatrales más famosas, A puerta cerrada (Sartre 2004, p. 93). De esta forma, las relaciones intersubjetivas se convierten en un vacío e, incluso, en una agresión. Beckett recoge esta determinación acerca del prójimo como un elemento esencial de su teatro.

En el caso de Albert Camus, también filósofo y escritor de la línea existencialista, el absurdo proviene del hecho de considerar -como Heidegger- que el hombre y la mujer siempre están enfrentados a la muerte; este destino igualador es, en realidad, una condena hacia la destrucción. Para Camus existe una pasión esencial, el absurdo. La ausencia de Dios en un mundo insensato establece una metafísica de lo irracional. Este desgarramiento frente a la existencia supone una destrucción tanto de los valores como de los mecanismos psíquicos de autoafirmación; en otras palabras, conlleva el lanzar al ser humano hacia el frío vacío existencial y a la destrucción de la ética de la solidaridad. Pasión, elección, libertad, emoción, sentimiento tienen una relación especular con la nada, lo aplanado y lo intrascendente, y, al fin, con lo destructivo. De ahí que para Camus sólo existe un problema filosófico realmente importante: el suicidio. Precisamente, con estas palabras inicia su obra El mito de Sísifo:

No hay más que un problema filosófico verdaderamente serio: el suicidio. Juzgar si la vida vale o no vale la pena de vivirla es responder a la pregunta fundamental de la filosofía. Las demás, si el mundo tiene tres dimensiones, si el espíritu tiene nueve o doce categorías, vienen a continuación (Camus, 1968, p. 125).

Por este motivo, y a diferencia de lo que una aproximación superficial pudiera hacer suponer, el hombre de Camus es un rebelde, de ningún modo es un nihilista. Esta redefinición de la vida sin objetivo, sin certeza de lo posible no fue privativa de los existencialistas. En efecto, Teodoro W. Adorno, de la llamada Escuela de Frankfurt, desde presupuesto 
cercanos al marxismo, va a presentar una historia sin esperanza (Rodríguez, 2004). Con ello reafirma este sentimiento de vacío propio de la época.

Curiosamente, con ellos coincidía la propuesta realizada por Sören Kierkegaard varias décadas antes. Sin embargo, para este filósofo danés, aunque la existencia es absurda en sí misma, sí tiene una salida en una fe absoluta. La fe en Dios como salto al vacío le otorga, en último término, un sentido a la existencia personal; pero, es una existencia que remite a la búsqueda de la autenticidad radical y, en consecuencia, el rechazo de la existencia falsa que le impone la vida social -la máscara de la persona-; la cual solo puede sobrellevarse con una cierta ironía socrática (Kierkegaard, 1965). De esta forma, se presenta un insólito encuentro entre el pensamiento ateo del absurdo de Sartre, Camus, Heidegger, entre otros, y el existencialismo cristiano de la fe, pues para ambas posiciones el absurdo de la existencia desemboca en aquello que sobrepasa a la razón; en un caso la nada, en el otro Dios. Así, tanto la fe como la nada llegan a ser los territorios privilegiados de la decisión; el no heideggeriano es ahora el reino de la libertad. Heidegger supera este sentido de lo absurdo, otorgándole al ser humano la posibilidad de un encuentro (Heidegger, 2003, pp. 247-283). La muerte como horizonte definitivo, como acabamiento absoluto, no significa un sinsentido: el redescubrimiento del ser lo encamina hacia el rumbo primigenio. José Ferrater Mora indica que el absurdo es, en consecuencia, "aferrarse apasionadamente a lo improbable" (Ferrater Mora, 1999, p. 35). Y el existencialismo cristiano termina por reafirman una ética de la solidaridad humana con un fundamento trascendente.

En efecto, el mundo del absurdo es fundamentalmente una rebelión y, a la vez, una búsqueda de la libertad. Esta forma de concebir el humanismo centrado en un sujeto individual implica un compromiso con su propia decisión. Compromiso y elección que marcan los caminos de la libertad. Pero que también redefine la función de género, con una profunda indagación sobre la identidad femenina, como hace Simone de Beauvoir (1998) y también de la expoliación del Tercer Mundo, de los condenados de la tierra, según la conocida expresión de Franz Fanon (1969). Extranjeros y extranjeras, arrojados en un mundo que no eligieron, deben ahora ejercer su dramática libertad. Rodeados por la náusea, el hombre y la mujer se describen como ausentes de ilusiones, de esperanzas y de perspectiva (Maclntyre, 1972, pp.147-154).

\section{Beckett y el teatro del absurdo}

Ninguno de estos elementos faltará a la cita en el teatro de Samuel Beckett. Este polifacético escritor nació en Dublín en 1906 y falleció en París en 1989. Elaboró un teatro que se caracteriza por un lenguaje aparentemente privado de sentido. Las palabras oscilan entre la evasividad, la comicidad y la filosofía. Las acciones suelen ser grotescas y tanto el 
vestuario, las luces, el escenario y la utilería destacan los rasgos anamórficos. Obviamente, estos elementos no son propios del autor, sino que más bien, corresponden al complejo reto de la puesta en escena. Asimismo, los diálogos son ambiguos y los monólogos suelen ser cortos y contradictorios. Todos estos mecanismos crean un ambiente de irracionalidad, desplazamiento metafórico de los significados que pueden suscitar en el espectador sentimientos de desolación extravagante e hilarante a la vez. Enfrentarse a tales textos o a estas representaciones escénicas implica una disposición anímica que pone en suspenso el sentimiento previo y las concepciones convencionales. Asimismo, los elementos espacio-temporales adquieren una dimensión irreal. El tiempo se subsume en unos pocos efectos: un árbol con sus reducidas hojas, un tenue desgaste de la voz, algún adverbio. Tampoco el espacio es paradójicamente revelador: un escenario reducido, un campo limitado. Nada cambia, pero todo pasa.

Esta singular estética recoge un lenguaje parco, rigurosamente cuidado y un argumento microscópico, casi anoréxico. Como se indicó anteriormente, corresponde a un minimalismo existencialista. Sin embargo, este minimalismo en realidad oculta una compleja trama plena de significados susceptible de varias hermenéuticas posible. En ellas el poder y la impotencia, la amistad y la incomunicación, la seriedad de la tragedia y la risa de la comedia se contraponen de forma continua como imágenes en un espejo que rememoran a Buster Keaton y a Charles Chaplin. Es como una presentia in absentia, oculta pero inevitable. Jenaro Talens ha recogido muy bien estos aspectos:

Samuel Beckett definía su trabajo por oposición al de Joyce, afirmando que mientras este último tendía a la omnisciencia y omnipotencia, él trabajaba con la impotencia y la ignorancia, algo que en su opinión, no había sido explotado con anterioridad ... Todo este complejo proceso de escritura, al desarrollarse, de modo explícito, en torno a la impotencia, se inscribe dentro de un entramado de relaciones donde son elemento esencial las cuestiones de poder, dado que la impotencia como condición sólo existe en tanto inscritas en ese entramado. Un poder visto no como opuesto a ausencia de poder sino como parte de un mecanismo tal como analizado por el modelo binario hegeliano amo/esclavo o, mejor aún, por la noción foucaultiana de poder "inmanente"; un poder que está en todas partes no porque lo controle todo, sino porque proviene de y reside en todas partes (Talens, 1999, pp. 556-569).

Con palabras simples, de uso corriente, muy lejos del espíritu de la tragedia clásica, Beckett confronta la banalidad de la sociedad burguesa con el absurdo de la existencia. Esta inclinación hacia el lenguaje habitual es un impasse absoluto en la trascendencia: el reino de la distopía. ¿O será, más bien, una visión posapocalíptica? En todo caso, para Beckett consistirá en la muerte de la tragedia. Como afirmó el crítico literario George Steiner "es la casi imposibilidad de un intercambio verbal" (Steiner, 2013, p.75). De esta forma, las palabras no solo son insuficientes para expresar lo inexpresable, sino que son, en sí mismas 
una traición a lo expresable. En su obra Acto sin palabras, continúa con esta misma reflexión: el silencio es la verdad. En este caso, Beckett lleva al máximo su fascinación por la intuición (Beckett, 2006).

\section{Aún no llega, ¿llegará?}

Esperando a Godot puede ser considerada la obra maestra del teatro de Beckett. Fue escrita a fines de la década de 1940, pero se publicó por primera vez en 1952 en París. Su representación inaugural también ocurrió en París al año siguiente. El mismo Beckett, autor bilingüe, realizó la traducción al inglés en 1954. Ha sido estimada justamente como una obra emblemática de la literatura y de la representación dramática y un hito teatral. Una obra imprescindible que contribuyó a redefinir el teatro a partir del siglo XX (Eagleton, 2011, p.105)

La trama es muy conocida. Obra de hora y media de duración en dos actos y con cinco personajes, posiblemente vagabundos o refugiados de la guerra: Estragón (Gogo), Vladimir (Didi), el cruel Pozzo y su esclavo Lucky (Afortunado) a quien controla con una cuerda, y un Muchacho que trae el mensaje de que Godot no vendrá hoy, pero sí mañana, un continuo mañana. Zapatos, sombreros, abrigos..., y en el fondo un árbol. "Camino en el campo, con árbol. Anochecer" (Beckett, 2006, p. 125) dice la indicación espacial y temporal del primer acto. En el segundo: "Al día siguiente. Misma hora. Mismo lugar ... El árbol tiene algunas hojas" (Beckett 2006, p. 170). Son detalles, los justos detalles, cuidadosamente escogidos por el autor para dar la sensación de realidad. Pero más que detalles, son propiamente elementos arquetípicos que conmueven la psique del lector y del espectador creando profundas resonancias inconscientes (Rodríguez, 2009, pp. 67-70). Es cierto, ya el árbol tiene algunas hojas, pocas, muy pocas. Pero hay más, mucho más.

Una observación de Umberto Eco se ajusta a este teatro: "para leer el mundo y los textos sospechosamente, es necesario haber elaborado algún tipo de método obsesivo" (Eco, 2013, p. 60). Esta afirmación supone una lectura guiada por la sospecha. Aquí sospecha no implica rechazo, sino más bien una lectura atenta para recoger los indicios mínimos, pero de una extraordinaria importancia que el autor va presentando progresivamente en su obra. Ya de antemano el espectador sabe que cada movimiento del cuerpo y el vestuario, cada cambio en la entonación, cada uno de los gestos, la luz, los espacios y, sobre todo, los silencios delatan una interacción semántica compleja. Entramado de ideas sugeridas, sentimientos ocultos y juegos de lenguaje. El minimalismo en la representación teatral y en literatura escrita implica que el lector-espectador esté muy atento al desarrollo de la obra; un lector que lee con sospecha. Requiere una actitud como la de un detective que recela de todos y de todo pues cada indicio es, al final, una prueba. 
Muchas lecturas son posibles. En un entorno social marcado por la desolación física y moral, el grito nietzscheano de la muerte de Dios adquiere un eco vital. Ya desde el título provocativo Beckett intenta crear resonancias muy propias de la cultura occidental, particularmente la europea. No tiene sentido preguntarse quién es Godot. Más bien cómo suena el nombre: God, Gott, Go-to: Fonema también de profundas resonancias inconscientes. Y Beckett sugirió que se derivaba de godillot, que en jerga del francés significa bota. El mismo Beckett había advertido en contra de las interpretaciones teológicas. Sin embargo, la obra posee frecuentes intertextos y referencias religiosas: la Biblia, el Cielo, el Infierno, etc. ¿Será que la ausencia de Dios implica la suprema soledad, la íntima incomunicación? El famoso monólogo y declamación de Lucky hacia la mitad del primer acto es una fuerte referencia a este tema: Dios es ahora un tema trivial. Es indigno de consideración seria. Son solamente palabras sinsentido (Beckett, 2006, pp. 158-160). En este monólogo y, aun en toda la obra, se percibe la subyacente influencia de la filosofía de Berkeley, para quien el mundo cobra existencia al ser percibido: "ser es ser percibido" nos ha dicho el filósofo inglés; marcando con ello un relativismo epistemológico y una extrema filosofía inmaterialista de la subjetividad (Berkeley, 2013, pp. 208-215). Porque en la tragedia de la incomunicación humana, percibir es ser en este diálogo-monólogo de sordos. O, más bien, como en Sartre, creer que se percibe es la razón de esta sinrazón comunicativa. Los antihéroes de esta obra están muy lejos de los héroes trágicos, Beckett evita el sacrifico y la acción ineluctable impuesta por el destino (De Saint-Victor, 1952)

Aquí se encuentra, como en un anverso, un concepto trascendental que se diluye en lo intrascendente. Se cierra dentro de un círculo impotente: una vista metafísica que se reduce a "iLas piedras!... ¡Tan tranquilas!" Sentimiento que recuerda al Darío de Lo fatal: "Dichoso el árbol que es apenas sensitivo..., / y más la piedra dura porque ésa ya no siente" (Darío, 2016, p. 154). Así, lo vital y animado se transmuta en lo inanimado; en lo existente en sí mismo como mónadas imposibles de comunicación. Soledad en compañía; posiblemente la peor de todas las soledades. Pero, a la vez, imposibilidad de separación: "Vladimir: ¿Te he dejado alguna vez? Estragón: Me has dejado marchar" (Beckett, 2006, p. 171). Tampoco será necesario pensar: el sombrero está en el suelo, ¿será para que ya no piense más? Y, entonces, mirar al cielo sin estrellas: "Vladimir: El fondo no cambia". Aquí no existe el salto al vacío de la fe de Kierkegaard; tampoco el hado de Sófocles. El ideal clásico es demasiado artificial para Beckett. Es interesante anotar que cuando se contraponen los dos actos se advierte una imagen especular, inversa: parece lo mismo, pero en realidad, todo es diferente. Pero en el segundo acto, Pozzo se ha vuelto ciego y Lucky mudo. Ceguera y mudez: oscuridad y silencio, ¿incomunicación, la nada...?

De nuevo, en este sutil juego de espejos, las contradicciones de las relaciones humanasy de la existencia se magnifican en un pequeño escenario: comunicación-incomunicación, 
saber-ignorancia, seriedad-comicidad, amistad-separación, sentir-pensar, trascendencia e inmanencia, palabra-silencio. Aporía de una espera interminable. Pasa el tiempo y vuelve a esperar; pérdida de la esperanza. Una espera sin esperanza, quizás esta sea la paradójica emotividad de la obra. "Estragón: Nada ocurre, nadie viene, nadie se va. Es terrible" (Beckett, 2006, p.157). Pero siempre, día tras día, la espera interminable de Godot. El tiempo adquiere una dimensión densa, casi perceptible, como si fuera un personaje más. Un tiempo detenido, como el avanzar en la carretera cuando es imposible la marcha. Beckett lo ha dicho: "Ante ese monstruo o dios jánico, triple, ágil: el Tiempo -una condición de la resurrección por ser un instrumento de la muerte-" (Beckett, 2008, p. 63). No es el tiempo de los relojes derretidos de Dalí, más bien se acerca a la imagen del tiempo de los relojes sin manecillas de las películas de Ingmar Bergman. Tiempo con mayúscula para otorgarle la poderosa fuerza de la determinación. Tiempo que marca el elusivo camino de la existencia: el ser y el tiempo.

Por supuesto, nunca sabremos quién es Godot ni su importancia, ni tan siquiera se sabrá qué propósito tiene. Eso no es lo importante. Tampoco en esta obra hay salida hacia el aburrimiento; no se renuncia a lo finito, pues no hay infinitud posible. Es, como dice el título de otra obra teatral de Beckett, un final de partida. Pero un final en el cual se sabe que Godot no llegará hoy, "pero seguro mañana sí vendrá". Y siguiendo a Camus, aún queda el suicido como alternativa: "Vladimir: nos ahorcaremos mañana a menos que venga Godot" (Beckett 2006, p. 208). Alternativa que se ha venido insinuando, pero que ahora, será la posible cuerda sostenida en un árbol que tiene ya algunas hojas. Un mañana que no llega, un Godot ausente. De esta forma, la idea que presenta Beckett se ajusta la filosofía existencialista según la cual la vida no es una sucesión luminosa de aciertos o un camino que tiene un final explicativo o razonables. Más bien la vida no tiene ningún propósito. Es una especie de neblina eterna que nos rodea continuamente. $Y$ termina con uno de los finales más célebres del teatro:

\author{
Estragón: ¿Qué? ¿Nos vamos? \\ Vladimir: Súbete los pantalones. \\ Estragón: ¿Cómo? \\ Vladimir: Súbete los pantalones. \\ Estragón: ¿Qué me quite los pantalones? \\ Vladimir: Súbete los pantalones. \\ Estragón: Ah, sí, es cierto. \\ (Se sube los pantalones. Silencio) \\ Vladimir: ¿Qué? ¿Nos vamos? \\ Estragón: Vamos. (No se mueven). \\ (Beckett 2006, p. 208).
}




\section{Horizonte del alma}

Diagnóstico de una época. Valores universales. Sentido de lo imposible. Ausencia de Dios: incomunicación. Quizás esta obra ya no impresione radicalmente a una generación cuyas preocupaciones siguen otros derroteros: la crisis ecológica y el cambio climático, la globalización y la mundialización, las migraciones, la concentración de la riqueza y el aumento de la pobreza, el fantasma del desempleo, el terrorismo y el fanatismo religioso, la comunicación tecnológica; y más aún, en el mundo donde la libertad personal y la puesta en cuestión de diversas escalas de valores en competencia ejemplifican un escenario mundial distinto al que existía en la época en que se escribió y se estrenó por primera vez. Una idea de libertad diferente, una sucesión de decisiones aceleradas y marcadas por la vigilancia policial de la tecnología. Teatro de minorías, ciertamente, pero de minorías expectantes de lo diverso. En el teatro de Beckett existe una continua tensión, o más bien ambigüedad, entre las pretensiones filosóficas y al mismo tiempo un rechazo alérgico a las mismas. Este hecho marca sus diálogos y le otorga una compleja interrelación entre lo dicho y lo no dicho, la palabra y el silencio, y, a la vez, la ausencia y la presencia del cuerpo. Una indeterminación de lo indecible, insinuado, apenas sugerido, pero nunca ausente. Según Terry Eagleton en Beckett,

La existencia tiene la torpe compulsión del destino sin propósito ... Porque, el mundo de Beckett lo pueblan, por tanto, quienes están por debajo de lo trágico, quienes olvidan su gran momento, y no se levantan en sus ocasiones dramáticas ni pueden apelar a la retórica para exagerar con éxito, demasiado secos y vacíos para participar en un colorista combate teatral (Eagleton, 2001, p. 107).

Al final de estas reflexiones cabe recordar un elemento esencial: este texto, como todos, tiene su propio camino; algo así como un desideratum marcado por su época, por supuesto, pero lanzado hacia el futuro. Si bien cada obra es hija de su tiempo, tiene también su propio destino. El escritor argentino Ricardo Piglia lo ha señalado con acierto:

Es decir que más allá de las redes sociales que se construyen, más allá de la presencia de la cultura de masas y los criterios de lectura, creo que los textos tienen su propia dinámica y son capaces de hacer un camino, más allá de cómo sabemos, lo que en verdad decide la consagración, el canon y la historia literaria, son redes no ligadas inmediatamente a la producción misma (Piglia, 2016, p. 141)

De tal modo, toda obra posee un carácter propio. Una determinación temporal que, en el caso de Esperando a Godot, la espera aún continúa. Y, como ha advertido la estética de la recepción, al fin y al cabo, es el propio lector y espectador quien articula el significado de la obra (Warning, 1989). Camino particular y hermenéutica propia: si la acción y la palabra son los soportes esenciales del texto, en consecuencia, en esta obra el espectador debe estar inmerso en la dimensión interna de los personajes debido a la casi carencia de 
acción. Una tensión psíquica que implica una profundización en los caracteres para insinuar otra dimensión poco más o menos metafísica. Una obra de engañosa simplicidad. Profunda y reflexiva. Indispensable en el teatro. Así como Sófocles o Eurípides, Shakespeare, Ibsen, García Lorca, Brecht, o...., cada generación tiene el derecho y el deber de presenciarla. Como ha señalado Kaufmann: "Infierno, Purgatorio, y Cielo no son para nosotros, exceptuando el hecho de que los tenemos aquí y ahora, en esta tierra. Los grandes poetas trágicos conocieron las tres cosas, y sus visiones pueden iluminar nuestro infierno" (Kaufmann, 1978, p. 21). El buen teatro, como toda buena literatura, nos hace más humanos. Es una ventana solidaria a la profundidad del alma.

\section{Referencias}

Beckett, S. (2008). Proust y otros ensayos. Santiago, Chile: Universidad Diego Portales.

Beckett, S. (2006). Teatro reunido. Barcelona: Tusquets Editores.

Benjamin, W. (2013). Libro de los pasajes. Madrid: Akal.

Berkeley, G. (2013). Tratado sobre los principios del conocimiento humano. Madrid: Gredos, S. A.

Camus, A. (1968). El mito de Sísifo. En Obras completas. Madrid: Aguilar.

Cicerón, M. T. (2002). Sobre el orador. Madrid: Gredos, S. A.

Darío, R. (2016). Del símbolo a la realidad. Obra selecta. Barcelona: Penguin Random House Grupo Editorial, S.L.U.

De Beauvoir, S. (1998). El segundo sexo. Madrid: Cátedra.

De Saint-Victor, Paul. (1952). Las dos carátulas. Historia del teatro griego y de las grandes épocas del arte teatral. Buenos Aires: El Ateneo Editorial.

Eagleton, T. (2011). Dulce violencia. La idea de lo trágico. Madrid. Trotta, S. A.

Eco, U. (2013). Interpretación y sobreinterpretación. Madrid: Akal

Fanon, Franz. (1969). Los condenados de la tierra. México: Fondo de Cultura Económica.

Ferrater-Mora, J. (1999). Diccionario de filosofía. Barcelona: Ariel.

Heidegger, M. (2000). Carta sobre el humanismo. En Martín Heidegger. Hitos. Madrid: Alianza Editorial, S.A.

Heidegger, M. (2003). El ser y el tiempo. Trad. Jorge Eduardo Rivera. Madrid: Trotta.

Kandel, E. R. (2012). The Age of Insight. From Vienna 1900 to the Present. Nueva York: 
Random House, Inc.

Kaufmann, W. (1978). Tragedia y filosofía. Barcelona: Seix-Barral, S. A.

Kierkegaard, S. (1965). El concepto de la angustia. Madrid: Guadarrama, S. A.

Lamana, M. (1967). Existencialismo y literatura. Buenos Aires: Centro Editor de América Latina, S. A.

MacIntyre, A. (1972). Existentialism. En Paul Edwards (Ed.), The Encyclopedia of Philosophy (Vol. 3). New York: Macmillan Publishing Co. Inc. \&The Free Press.

Moretti, F. (1996). Modern Epic. The World System from Goethe to García Márquez. London-New York: Verso.

Nietzsche, F. (2012). El nacimiento de la tragedia o helenismo y pesimismo. Madrid: Valdemar.

Nussbaum, M. C. (2008). Paisajes del pensamiento. Barcelona: Paidós Ibérica, S.A.

Piglia, R. (2016). La forma inicial. Conversaciones en Princeton. San José: Ediciones Lanzallamas.

Rodríguez Zamora, J. M. (2002). El ser en la palabra; sobre el texto poético como filosofía. Revista de Filología y Lingüística, XXVIII(I), 57-67.

Rodríguez Zamora, J. M. (2004). La negación de la esperanza: dialéctica negativa y experiencia religiosa en T.W. Adorno. Revista de Filosofía de la Universidad de Costa Rica, $X L I /(105), 1001-1110$.

Rodríguez Zamora, J. M. (2009). El héroe. Literatura y psicología analítica. Revista de Filología y Lingüística, XXXV(I), 65-86.

Schopenhauer, A. (2004). El mundo como voluntad y representación. Madrid: Trotta.

Segura Munguía, S. (1985). Diccionario etimológico latino-español. Madrid: Ediciones Generales Anaya

Sartre, J. P. (2004). A puertas cerradas / La puta respetuosa. Buenos Aires: Losada

Sartre, J. P. (1998). El existencialismo es un humanismo. Buenos Aires: Losada. 1998.

Sartre, J. P. (1966). El ser y la nada. Ensayo de ontología fenomenológica. Buenos Aires: Losada, S. A.

Steiner, G. (2013). Lenguaje y silencio. Ensayos sobre la literatura, el lenguaje y lo inhumano. Barcelona: Gedisa.

Talens, J. (2006). Signifique quien pueda o La voz de qué amo. En Samuel Beckett, Teatro reunido. Barcelona: Tusquets Editores.

Warning, R. (Ed.) (1989). Estética de la recepción. Madrid: Visor, S. A. 